PUPT-1528

hep-th/9503014

\title{
Magnetic Fields and Fractional Statistics in Boundary Conformal Field Theory
}

\author{
C. G. Callan, I. R. Klebanov, J. M. Maldacena and A. Yegulalp \\ Department of Physics, Princeton University \\ Princeton, NJ 08544
}

\begin{abstract}
We study conformal field theories describing two massless one-dimensional fields interacting at a single spatial point. The interactions we include are periodic functions of the bosonized fields separately plus a "magnetic" interaction that mixes the two fields. Such models arise in open string theory and dissipative quantum mechanics and perhaps in edge state tunneling in the fractional quantized Hall effect. The partition function for such theories is a Coulomb gas with interchange phases arising from the magnetic field. These "fractional statistics" have a profound effect on the phase structure of the Coulomb gas. In this paper we present new exact and approximate results for this type of generalized Coulomb gas.
\end{abstract}




\section{Introduction}

Free field theories in $1+1$ dimensions with boundary interactions of various types have been the subject of much recent work. Such systems describe impurity scattering [1], tunneling between quantum Hall edges [2], dissipative quantum mechanics [3, [4] and, in particle physics, open strings in background fields [5, 6, 7] and monopole catalysis [8]. These theories are often soluble since all the nontrivial dynamics are localized at a single spatial point and amount to a dynamical boundary condition on otherwise free fields. As usual, an important problem is that of finding the conformally-invariant fixed points to which these theories flow in the infrared (often the limit of most physical interest). Even for the simple case of a single free scalar field, it turns out that the class of conformally invariant boundary conditions is much larger than the familiar Dirichlet and Neumann conditions [9]. In this paper we will present a new example, interesting both for its possible practical applications and for the theoretical issues it raises, in which the conformal field theory is exactly soluble.

We will be concerned with the theory of two massless scalar fields on a half line, with boundary interactions generated by a periodic potential for each scalar field separately and by a magnetic field term which mixes the two fields. This system describes in a very direct way the dissipative quantum mechanics (DQM) of an electron moving in two dimensions subject to a magnetic field and a square lattice potential (the Wannier-Azbel-Hofstadter model [10]). By tuning the period of the potentials and the strength of the magnetic field one can reach $c=2$ conformal theories corresponding to phase transitions between localized and delocalized long-time behavior of the electron. From the point of view of string theory, the conformal fixed points identify spacetime field configurations that solve the classical open string field equations.

In this paper we will construct such $c=2$ boundary conformal field theories. Our results are a logical extension of our previous work [9] on the $c=1$ problem of a single scalar field subject to a boundary periodic potential (and no magnetic field, of course). There we found that if the period of the potential is chosen such that the boundary interaction has naive dimension one, the theory is conformal for any potential strength. The conformal boundary state, which summarizes the quite non-trivial scattering of asymptotic particles from the boundary interaction, turns out to be a global $S U(2)_{L}$ rotation (with rotation angle set by the strength of the potential) of the non-interacting boundary state. The partition functions and S-matrix elements of the critical theory are accordingly simple and explicitly calculable. 
The $c=2$ theory, the subject of this paper, is more complex and more interesting. First, the magnetic field affects the dimension of the potential operators. The condition that this dimension be unity defines, to lowest order in the potential strength, a circle in the plane of magnetic field strength, $\beta$, and strength of dissipation, $\alpha$ (this parameter can also be thought of as controlling the period of the potential). The interesting complexity comes in when we ask what happens as the potential strength is turned on. We find that at certain isolated points along this circle ("magic" values of the magnetic field and dissipation strength), the situation is much like the $c=1$ case: the dimension of the potential operator is unaffected by the strength of the interaction and the boundary state can be explicitly constructed out of $S U(2)_{L}$ actions on the non-interacting boundary state. At generic values of $\alpha$ and $\beta$, however, there are subleading corrections to the beta function which cause a renormalization group flow of the potential strength. For $\alpha, \beta$ near the critical circle the potential strength $V$ flows to a perturbatively reliable infrared stable fixed point at small $V_{c}$. These fixed points should have quite interesting physics which can be explored by perturbative methods.

The theoretical framework for all of this is the one-dimensional Coulomb gas. When the partition function for theories of the type we are discussing is expanded in the potential strength, each insertion of a periodic potential behaves like a charge interacting with other charges via a logarithmic potential. The $c=1$ theory describes a gas of + and - charges, with all orderings of the charges contributing with equal weight. The $c=2$ case corresponds to a Coulomb gas with two flavors $(X$ and $Y$ ) of charge. The two flavors are independent, except for a phase factor $e^{i q_{X} q_{Y} \phi}$ associated with the interchange of an $X$ and a $Y$ charge, where $q_{X}$ and $q_{Y}$ are the signs of the charges, and $\phi$ depends on $\alpha$ and $\beta$. Many interesting effects have to do with these "fractional statistics" of the Coulomb gas (the "magic" points correspond to phases which are integral multiples of $2 \pi$, so that the two flavors become independent). This Coulomb gas with "fractional statistics" has received little attention to date, but, as we hope this paper will demonstrate, it is worth investigating as it may underlie some systems of real practical interest.

\section{Setup and Renormalization Group Analysis}

We will be considering a system of two massless scalar fields in $1+1$ dimensions. The fields are free in the bulk but have certain boundary interactions. Specifically, we take the 
action to be $S=S_{b u l k}+S_{\text {pot }}+S_{\text {mag }}$ where

$$
\begin{aligned}
S_{b u l k} & =\frac{\alpha}{4 \pi} \int_{0}^{T} d t \int_{0}^{l} d \sigma\left(\left(\partial_{\mu} X\right)^{2}+\left(\partial_{\mu} Y\right)^{2}\right), \\
S_{\text {pot }} & =V \int_{0}^{T} d t(\cos X(0, t)+\cos Y(0, t)), \\
S_{m a g} & =i \frac{\beta}{4 \pi} \int_{0}^{T} d t\left(X \partial_{t} Y-Y \partial_{t} X\right)_{\sigma=0} .
\end{aligned}
$$

The parameters $\alpha$ and $\beta$ are defined as in [11]: $\alpha$ determines the strength of dissipation in DQM (or, if we rescale the fields, the period of the interaction potential); $\beta$ is related to the magnetic field normal to the $X-Y$ plane via $\beta=2 \pi B$. We have normalized the fields so that the period of the boundary potential is $2 \pi$. At this point we have chosen to put the boundary interactions on the $\sigma=0$ boundary and to leave the $\sigma=l$ boundary free, but, depending on the application, we might wish to put interactions on the other boundary as well or even move the second boundary to infinity in order to discuss scattering.

Comparing with the action of the $c=1$ theory [9], we note that the truly new feature of the $c=2$ system is $S_{m a g}$. This term introduces a boundary coupling between $X$ and $Y$ without destroying the conformal invariance. Indeed, for $V=0$, the theory is free and obviously conformally invariant for any $\alpha$ and $\beta$. Turning on the potential in general spoils the conformal invariance, but we will find that it is preserved on some surfaces in the $(\alpha, \beta, V)$ space. The theory turns out to be especially simple at the so-called "magic" parameter values,

$$
(\alpha, \beta)=\left(\frac{1}{n^{2}+1}, \frac{n}{n^{2}+1}\right), \quad n \in \mathbb{Z}
$$

where it is conformally invariant for any potential stength $V$. Since the magnetic flux per unit cell of the potential lattice is $2 \pi \beta$, the "magic" points do not correspond to trivial fluxes (although they do correspond to trivial interchange phases of the Coulomb gas). Thus, these physically non-trivial points possess hidden simplicity which can be efficiently explored using boundary state techniques.

The theory defined by (2.1) makes perfect sense for non-compact fields $X$ and $Y$ (this is the physically interesting choice from the DQM point of view). In other applications we may define $X$ and $Y$ on circles of radius $R$. The periodicity of the potential constrains $R$ to be a positive integer. A further constraint on the parameters arises from the singlevaluedness of the path integral. Under a physically unobservable shift $X \rightarrow X+2 \pi R$,

$$
\Delta S_{m a g}=i \beta R \int_{0}^{T} d t \partial_{t} Y=2 \pi \beta R^{2}
$$


where we take $Y$ to wind once around the target space. For the theory to be well-defined, we require $\Delta S_{\text {mag }}=2 \pi n$, which implies $\beta=n / R^{2}$. This is just the usual flux quantization condition. Since both $R$ and $n$ are integers, only rational values of $\beta$ are admissible in compactified theories.

We shall want to compute the functional integral for this system. As is well-known [12], it can be regarded either as an open string partition function $Z^{B_{V} N}=\operatorname{tr}\left(e^{-T H_{\text {open }}}\right)$ or as the amplitude for free closed string propagation between two boundary states $Z^{B_{V} N}=$ $\left\langle B_{V}\left|e^{-l\left(L_{0}+\tilde{L}_{0}\right)}\right| N\right\rangle$. In these expressions, the superscripts denote the boundary conditions at the ends of the open string: $|N\rangle$ is the Neumann boundary state and $\left|B_{V}\right\rangle$ is the boundary state induced by the magnetic and potential interactions. Since the magnetic boundary action taken by itself is exactly soluble [6], it is useful to express $\left|B_{V}\right\rangle$ as the magnetic boundary state, $\left|B_{0}\right\rangle$, acted on by the potential term in the path integral. This gives a more explicit expression for the partition function on the cylinder of circumference $T$ and length $l$ :

$$
Z^{B_{V} N}=\left\langle B_{0}\left|e^{-V \int_{0}^{T} d t[\cos X(0, t)+\cos Y(0, t)]} e^{-l\left(L_{0}+\widetilde{L}_{0}\right)}\right| N\right\rangle
$$

To calculate this object, we expand it in powers of $e^{ \pm i X}$ and $e^{ \pm i Y}$ :

$$
\begin{aligned}
& Z^{B_{V} N}=Z^{B_{0} N} \sum_{0}^{\infty} \frac{(-V / 2)^{n_{+}^{X}+n_{-}^{X}+n_{+}^{Y}+n_{-}^{Y}}}{n_{+}^{X} ! n_{-}^{X} ! n_{+}^{Y} ! n_{-}^{Y} !}\left\langle\prod_{j=1}^{n_{+}^{X}} \int_{0}^{T} d t_{j} e^{i X\left(0, t_{j}\right)} \times\right. \\
& \left.\prod_{j=1}^{n_{-}^{X}} \int_{0}^{T} d t_{j} e^{-i X\left(0, t_{j}\right)} \prod_{j=1}^{n_{+}^{Y}} \int_{0}^{T} d t_{j} e^{i Y\left(0, t_{j}\right)} \prod_{j=1}^{n_{-}^{Y}} \int_{0}^{T} d t_{j} e^{-i Y\left(0, t_{j}\right)}\right\rangle .
\end{aligned}
$$

The angle bracket denotes expectation value in the soluble theory with magnetic field on one boundary. This assigns a certain Gaussian propagator to the $X(0, t)$ and $Y(0, t)$ fields. The partition function then has the structure of a one-dimensional two-component plasma with the $e^{ \pm i X}$ and $e^{ \pm i Y}$ operator insertions acting as two distinct flavors of \pm charges.

We are mainly interested in finding the points in $(\alpha, \beta, V)$ space where these theories are conformal. The cylinder partition function (2.2) is a rather sensitive observable, but a simpler test of conformal invariance is provided by its $l \rightarrow \infty$ limit. In this limit, the cylinder becomes conformally equivalent to a disk and the partition function reduces to

$$
Z_{\text {disk }}=\left\langle B_{V} \mid 0\right\rangle=\left\langle B_{0}\left|e^{-V \int_{0}^{T} d t[\cos X(0, t)+\cos Y(0, t)]}\right| 0\right\rangle
$$


where $T$ is the circumference of the outer boundary of the disk (it will serve as an infrared cutoff for our calculations). Its perturbative expansion is still given by the Coulomb gas formula (2.3) and the Green functions needed to evaluate it can be shown to have the following simple forms [11]:

$$
\begin{aligned}
& \langle X(t) X(0)\rangle=\langle Y(t) Y(0)\rangle=-\frac{\alpha}{\alpha^{2}+\beta^{2}} \ln \left(4 \sin ^{2} \frac{\pi t}{T}\right) \\
& \langle Y(t) X(0)\rangle=\frac{i \pi \beta}{\alpha^{2}+\beta^{2}}(-1+2 \operatorname{frac}(t / T))
\end{aligned}
$$

where $0 \leq \operatorname{frac}(x)<1$ is the fractional part of $x$. The $X$-charges (and the $Y$-charges) interact with themselves via the usual Coulombic logarithmic potential, but the magnetic field induces a peculiar interaction phase between different types of charge. Since frac $(x)$ jumps by unity every time $x$ passes through an integer, the phase of the amplitude jumps by $2 \pi \beta /\left(\alpha^{2}+\beta^{2}\right)$ when the time ordering of an $X$ and a $Y$ charge is interchanged. The smooth linear variation of the phase between such jumps actually cancels out in neutral amplitudes (the only ones of concern to us). Thus, the entire effect of the $X Y$ interaction is summarized by weighting different orderings of the $X$ and $Y$ charges with appropriate phases: fractional statistics in one dimension.

It is now fairly straightforward to work out the first two terms in the renormalization group flow equation for the potential $V$. The first term, coming from normal ordering a single insertion of the potential, reads

$$
\frac{d V}{d \tau}=\left(1-\frac{\alpha}{\alpha^{2}+\beta^{2}}\right) V, \quad \tau=\log (T / \epsilon)
$$

where $T$ is the disk circumference and $\epsilon$ is an ultraviolet cut-off. In other words, the effective dimension of the boundary potential is $h=\alpha /\left(\alpha^{2}+\beta^{2}\right)$ and, to leading order, the theory is conformally invariant on the circle $h=1$. In the $c=1$ case the leading order condition for conformal invariance turned out to be exact. In order to study whether the same is true in the $c=2$ case, we need to calculate corrections to the beta function. Techniques for such calculations were outlined in [13].

The next correction is of $O\left(V^{3}\right)$ and is extracted from the correction to matrix element of the $e^{i X(0)}$ operator coming from insertions of $e^{i Y\left(t_{1}\right)}$ and $e^{-i Y\left(t_{2}\right)}$ near $e^{i X(0)}$ (with subsequent integration over $t_{i}$ ). If this has a logarithmic divergence, we must interpret this as a correction of order $V^{3}$ to the beta function. Isolating the connected part of the 
correlation function, and including the phases that account for the different orderings of $e^{ \pm i Y}$ with respect to $e^{i X}$ we find, after mapping disk to upper half-plane, the integral

$$
\frac{V^{3}}{16 \pi^{2}}\left[\exp \left(\frac{2 \pi i \beta}{\alpha^{2}+\beta^{2}}\right)+\exp \left(-\frac{2 \pi i \beta}{\alpha^{2}+\beta^{2}}\right)-2\right] \int_{0}^{\infty} d t_{1} \int_{-\infty}^{0} d t_{2}\left(t_{1}-t_{2}\right)^{-2}
$$

which is easily seen to have a logarithmic divergence:

$$
\int_{0}^{\infty} d t_{1} \int_{-\infty}^{0} d t_{2}\left(t_{1}-t_{2}\right)^{-2} \rightarrow \int_{\epsilon}^{T} d t_{1} \frac{1}{t_{1}}=\log (T / \epsilon)
$$

The resulting corrected beta function equation is

$$
\frac{d V}{d \tau}=V\left(1-\frac{\alpha}{\alpha^{2}+\beta^{2}}\right)-\frac{V^{3}}{4 \pi^{2}} \sin ^{2}\left(\frac{\pi \beta}{\alpha^{2}+\beta^{2}}\right)+\mathcal{O}\left(V^{5}\right)
$$

Now that we have computed the flow equation (2.4), we can study the fixed points. There is a class of "trivial" fixed points where the beta function vanishes order by order in $V$. The first term vanishes everywhere on the $h=1$ circle, while the second term vanishes only at the discrete points where $\frac{\beta}{\alpha^{2}+\beta^{2}}$ is integer as well. These are precisely the "magic" points where the interchange phases between the $X$ and $Y$ charges become trivial. On a disk, then, the $X$ and $Y$ charges no longer interact, so that the partition function reduces to a product of two $c=1$ partition functions with marginal boundary potentials. The exact conformal invariance of such $c=1$ theories was demonstrated in [9, 14]. This implies that at the "magic" points on the $h=1$ circle the beta function indeed vanishes order by order in $V$.

While the "magic" points are trivial fixed points for any $V$, the rest of the $h=1$ circle has no true fixed points except for the free theory with $V=0$. Consider, however, values of $\alpha$ and $\beta$ slightly outside the circle: (2.4) shows that, for $h=\alpha /\left(\alpha^{2}+\beta^{2}\right)$ slightly less than $1, V$ flows to an infrared stable fixed point at

$$
V_{c}^{2}=4 \pi^{2}(1-h) / \sin ^{2}\left(\frac{\pi \beta}{\alpha^{2}+\beta^{2}}\right) .
$$

The parameters $\alpha$ and $\beta$ are not renormalized and may be chosen so that $V_{c}$ is small and the perturbative argument is reliable. A most interesting feature of these non-trivial fixed points is that the flow starts with a perturbatively relevant operator of dimension $h<1$, which destabilizes the trivial fixed point at $V=0$. The effect of our interchange phases is to turn a naively massive theory into a conformal one! Could there be an experimental realization for this? 
The picture of the critical surface in $(\alpha, \beta, V)$ space which emerges from this is quite interesting. It is easiest to describe in terms of slices through the surface at constant $V$. For $V=0$ we find the circle $\alpha /\left(\alpha^{2}+\beta^{2}\right)=1$. As we increase $V$, the circle bulges outwards, while staying pinned at the "magic" points. In next-to-leading approximation, the critical circle deforms to the curve

$$
\beta(\alpha)^{2}=\frac{1}{4}-\left(\alpha-\frac{1}{2}\right)^{2}+\frac{V^{2}}{4 \pi^{2}} \alpha \sin ^{2}\left(\pi \sqrt{\frac{1-\alpha}{\alpha}}\right) .
$$

It is not obvious from these arguments what happens as $V$ gets large, but some sort of perturbation theory in $1 / V$ ought to be possible since, in that limit, one is approaching the trivial Dirichlet boundary condition.

There are two instructive consistency tests to be made at this point. First in open string theory, it is legitimate to regard the beta function as the equation of motion following from an action function given by the disk partition function itself: $S_{\text {eff }}=\ln Z_{\text {disk }}$. The $V$-independent piece, the disk amplitude in the presence of a boundary magnetic field, was calculated a long time ago [6,7] (it is the Born-Infeld action). If we introduce independent potential strengths for $\cos X$ and $\cos Y, V_{X}$ and $V_{Y}$, then there is a quadratic term given by

$$
\frac{V_{X}^{2}+V_{Y}^{2}}{4} \int_{0}^{2 \pi} \frac{d y}{2 \pi}[2 \sin (y / 2)]^{-2 h}
$$

where $h=\alpha /\left(\alpha^{2}+\beta^{2}\right)$ is the dimension of the potential operators, and we have set the circumference $T$ of the disk to unity. This integral can be calculated [15], giving

$$
\frac{V_{X}^{2}+V_{Y}^{2}}{4} \frac{\Gamma(1-2 h)}{\Gamma^{2}(1-h)}=\frac{V_{X}^{2}+V_{Y}^{2}}{8}\left[h-1+\mathcal{O}\left((h-1)^{2}\right)\right]
$$

where the right hand side is an approximation valid near the $h=1$ circle. The quartic terms have been calculated for other reasons in [11] and, when added to the lower-order terms give, in the vicinity of the $h=1$ circle,

$$
\ln Z_{\text {disk }}=\frac{1}{2} \ln \left[1+(\beta / \alpha)^{2}\right]+\frac{V_{X}^{2}+V_{Y}^{2}}{8}\left[\frac{\alpha}{\alpha^{2}+\beta^{2}}-1\right]+\frac{V_{X}^{2} V_{Y}^{2}}{32 \pi^{2}} \sin ^{2}\left(\frac{\pi \beta}{\alpha^{2}+\beta^{2}}\right) .
$$

Interpreting this as an effective action for $V_{X}$ and $V_{Y}$, varying with respect to $V_{X}$ and setting $V_{X}=V_{Y}$, we recover precisely the beta function (2.4)!

Second, recall that in theories with boundary interactions, $\ln Z_{\text {disk }}$ is the analogue of the Zamolodchikov $c$-function and is believed to decrease along the renormalization group 
flow [16]. It is not hard to check that this is the case for our example. In the UV theory with $V_{X}=V_{Y}=0, \ln Z_{\text {disk }}=\frac{1}{2} \ln \left[1+(\beta / \alpha)^{2}\right]$. It is also true that

$$
\frac{\partial \ln Z_{\text {disk }}}{\partial V_{X}}<0, \quad \frac{\partial \ln Z_{\text {disk }}}{\partial V_{Y}}<0,
$$

for $0<V_{X}, V_{Y}<V_{c}$, which implies that $\ln Z_{\text {disk }}$ indeed decreases along the trajectories. At the IR fixed point with $V_{X}=V_{Y}=V_{c}$ it reaches the value

$$
\ln Z_{\text {disk }}=\frac{1}{2} \ln \left[1+(\beta / \alpha)^{2}\right]-\left[\frac{\alpha}{\alpha^{2}+\beta^{2}}-1\right]^{2} \frac{\pi^{2}}{2 \sin ^{2}\left(\frac{\pi \beta}{\alpha^{2}+\beta^{2}}\right)}
$$

to the order we have retained.

\section{Exact Boundary States at "Magic" Magnetic Fields}

In the previous section we showed that at certain isolated points (where $\alpha=\alpha^{2}+\beta^{2}$ and $\beta / \alpha$ is integer) the theory is exactly conformal order by order in an expansion in the potential strength. We will now show how to reduce the calculation of the boundary state at these points to an algebraic exercise. Once the boundary state is known, any other quantity of physical interest can be computed.

We start with the familiar oscillator expansion of the pure magnetic field boundary state [6]:

$$
\begin{aligned}
\left|B_{0}\right\rangle=\sqrt{1+(\beta / \alpha)^{2}} & \exp \left\{\sum _ { n > 0 } \frac { 1 } { n } \left(\tilde{\alpha}_{-n}^{X}\left(\cos \delta \alpha_{-n}^{X}+\sin \delta \alpha_{-n}^{Y}\right)+\right.\right. \\
& \left.\left.\tilde{\alpha}_{-n}^{Y}\left(\cos \delta \alpha_{-n}^{Y}-\sin \delta \alpha_{-n}^{X}\right)\right)\right\}|0\rangle .
\end{aligned}
$$

In this expression, the oscillators are defined by the usual expansion of the boundary field

$$
X(t, 0)=\sqrt{\frac{\alpha}{2}} \sum_{n \neq 0} \frac{1}{|n|}\left(\alpha_{n}^{X} e^{i 2 \pi n t / T}+\tilde{\alpha}_{n}^{X} e^{-i 2 \pi n t / T}\right)
$$

and the reflection phase $\delta$ is defined by

$$
\sin \delta=\frac{2 \alpha \beta}{\alpha^{2}+\beta^{2}} \quad \text { or } \quad \tan \frac{\delta}{2}=\frac{\beta}{\alpha} .
$$

Using this definition of $\delta$, the overall normalization factor of $\sqrt{1+(\beta / \alpha)^{2}}$ can be rewritten as $\sec (\delta / 2)$ and will be so written in all further appearances of the boundary state. The net effect of the magnetic field is a chiral $O(2)$ rotation of the Neumann boundary state:

$$
\left|B_{0}\right\rangle=\sec (\delta / 2) e^{i \delta \mathcal{R}_{L}}|N\rangle
$$


where

$$
\mathcal{R}_{L}=\left(y_{L}^{0} p_{L}^{X}-x_{L}^{0} p_{L}^{Y}\right)+\sum_{n>0} \frac{i}{n}\left(\alpha_{n}^{Y} \alpha_{-n}^{X}-\alpha_{-n}^{Y} \alpha_{n}^{X}\right)
$$

(We have included the zero mode parts for completeness, but they do not contribute since the Neumann state on which it acts has zero momentum). The interacting boundary state is obtained from the above by acting on it with the boundary potential term:

$$
\left|B_{V}\right\rangle=\sec (\delta / 2) e^{-H_{\text {pot }}(X)-H_{\text {pot }}(Y)} e^{i \delta \mathcal{R}_{L}}|N\rangle .
$$

where $H_{\text {pot }}(X)=\int d t V \cos X(t, 0)$.

We now show how to relate this to the explicitly known $c=1$ boundary state of [9]: First, move the $O(2)$ rotation to the left to get

$$
\begin{aligned}
e^{i \delta \mathcal{R}_{L}} e^{-i \delta \mathcal{R}_{L}} & e^{-H_{p o t}(X)-H_{p o t}(Y)} e^{i \delta \mathcal{R}_{L}}|N\rangle= \\
& e^{i \delta \mathcal{R}_{L}} e^{\left\{e^{-i \delta \mathcal{R}_{L}}\left(-H_{p o t}(X)-H_{p o t}(Y)\right) e^{i \delta \mathcal{R}_{L}}\right\}}|N\rangle .
\end{aligned}
$$

Since the $O(2)$ generator acts only left-movers, we must break fields up into their chiral components in order to evaluate the rotated potential term:

$$
e^{-i \delta \mathcal{R}_{L}} H_{p o t}\left(X_{L}+X_{R}\right) e^{i \delta \mathcal{R}_{L}}=H_{p o t}\left(\cos \delta X_{L}-\sin \delta Y_{L}+X_{R}\right)
$$

Since this operator acts directly on a Neumann boundary state, we can use the Neumann boundary condition $X_{L} \rightarrow X_{R}$ to reduce this to

$$
H_{p o t}\left(\cos \delta X_{L}-\sin \delta Y_{L}+X_{L}\right)=H_{p o t}\left(2 \cos \delta / 2\left(\cos \delta / 2 X_{L}-\sin \delta / 2 Y_{L}\right)\right)
$$

A similar expression gives the effect of the rotation on $H_{p o t}(Y)$. To simplify further still, we define rotated and rescaled coordinates

$$
X^{\prime}=\cos \delta / 2(\cos \delta / 2 X-\sin \delta / 2 Y), \quad Y^{\prime}=\cos \delta / 2(\cos \delta / 2 Y+\sin \delta / 2 X)
$$

in terms of which the boundary state becomes

$$
\left|B_{V}\right\rangle=\sec (\delta / 2) e^{i \delta \mathcal{R}_{L}} e^{-H_{p o t}\left(2 X_{L}^{\prime}\right)-H_{p o t}\left(2 Y_{L}^{\prime}\right)}\left|N^{X^{\prime}}\right\rangle\left|N^{Y^{\prime}}\right\rangle
$$

We have written the Neumann boundary state as the product of an $X^{\prime}$ and a $Y^{\prime}$ factor to emphasize that, apart from the $O(2)$ rotation, (3.4) has become the product of two $c=1$ boundary states. The rescaling of the fields changes the effective value of $\alpha$ in the bulk 
action (2.1) to $\tilde{\alpha}=\alpha / \cos ^{2} \delta / 2=\left(\alpha^{2}+\beta^{2}\right) / \alpha$. On the critical circle $\tilde{\alpha}=1$ and $H_{\text {pot }}\left(2 X_{L}^{\prime}\right)$ is the integral of a dimension-one chiral field. As a result, the $X^{\prime}$ and $Y^{\prime}$ factors in (3.4) become identical to the $c=1$ conformal boundary states constructed in [9]. Because the $O(2)$ rotation commutes with the Virasoro generators, (3.4) is guaranteed to satisfy the famous conformal boundary state condition $\left(L_{n}-\tilde{L}_{-n}\right)|B\rangle=0$ [17].

There are two subtleties to bear in mind: compactification and interchange phases. In performing the $X_{L} \rightarrow X_{R}$ replacement, various operators have to be commuted past each other and we have assumed that operators built out of orthogonal linear combinations of $X$ and $Y$ commute. This is true only for "magic" points of the magnetic field: as we discussed in the preceding section, for other values of the field, extra phases appear. Also, the above naive treatment of the breakup of operators into left- and right-movers is strictly valid only for fields compactified to the $S U(2)$ radius. The left and right moving momenta will depend on the compactification radius, and, for this particular radius only, left and right moving momenta are independent. For any radius, the Neumann state has zero total momentum $\left(p_{L}=-p_{R}\right)$ and contains only winding modes. For infinite compactification radius there are no winding modes (i.e. $p_{L}=p_{R}$ ) and the two conditions taken together imply that the $R=\infty$ Neumann state has $p_{L}=p_{R}=0$. A key point is that we can compute the infinite radius boundary state by first doing the computation at the $S U(2)$ radius (as above) and then inserting a projection $P_{\infty}$ onto states states with $p_{L}=p_{R}$ 目:

$$
\left|B_{V}\right\rangle=\sec (\delta / 2) P_{\infty} e^{i \delta \mathcal{R}_{L}} e^{-H_{p o t}\left(2 X_{L}^{\prime}\right)-H_{p o t}\left(2 Y_{L}^{\prime}\right)}|N\rangle_{S U(2)}
$$

In what follows, we will show how to make practical use of this formula.

Let us begin by studying the cylinder partition function between a Neumann and an interacting boundary at infinite compactification radius. Using the above transformation of the interacting boundary state, we can write

$$
Z^{N B_{V}}=\sec (\delta / 2)\left\langle N\left|q^{L_{0}+\tilde{L}_{0}} e^{i \delta \mathcal{R}_{L}} e^{-H_{p o t}\left(2 X_{L}^{\prime}\right)-H_{p o t}\left(2 Y_{L}^{\prime}\right)}\right| N\right\rangle
$$

The projector $\mathrm{P}_{\infty}$ onto $p_{L}=p_{R}$ disappears because the Neumann state $|N\rangle$ has zero left and right moving momenta. Also, as we have no operators that change the right moving momentum, only the zero momentum component of the state $|N\rangle_{S U(2)}$ contributes, leaving just the usual Neumann boundary state $|N\rangle$. We can perform the transformation

1 At a finite multiple of the $\mathrm{SU}(2)$ radius, $R=N \sqrt{2}$, the construction is similar but the projection is onto states that have the allowed winding number $p_{L}-p_{R}=k N / \sqrt{2}$. 
$X \rightarrow X^{\prime}, Y \rightarrow Y^{\prime}$ everywhere in the previous equation, since the Neumann states, the $\mathcal{R}_{L}$ operators and the $L_{0}, \tilde{L}_{0}$ operators are all invariant under rotations in the $X Y$-plane (the rotation affects the left- and right-movers in the same way ). The end result is a greatly simplified expression for the partition function:

$$
Z^{N B_{V}}=\sec (\delta / 2)\left\langle N\left|q^{L_{0}+\tilde{L}_{0}} e^{i \delta \mathcal{R}_{L}} e^{-H_{p o t}\left(2 X_{L}\right)-H_{p o t}\left(2 Y_{L}\right)}\right| N\right\rangle .
$$

The effect of the potential is to replace the Neumann boundary by a product of two interacting $c=1$ boundary states which are then mixed together in the partition function by the chiral $O(2)$ rotation $e^{i \delta \mathcal{R}_{L}}$. This simplification was possible because the $X$ and $Y$ potential operators effectively commute for the "magic" field values satisfying $\tan (\delta / 2)=$ $n$, with $n \in \mathbb{Z}$.

The fact that (3.6) is almost completely factorized into two exact $c=1$ boundary states enables us to obtain an explicit expansion of the partition function in powers of q. We exploit the fact that the $c=1$ boundary states are built on $\mathrm{SU}(2)_{1}$ Kac-Moody algebras. We actually have two commuting algebras, one for each boson, with the currents

$$
\begin{array}{cl}
J^{X \pm}(z)=e^{ \pm i 2 X(z)}, & J^{Y \pm}(z)=e^{ \pm i 2 Y(z)}, \\
J^{X 3}(z)=i \partial_{z} X(z), & J^{Y 3}(z)=i \partial_{z} Y(z) .
\end{array}
$$

Using the results of [9] on the expression of the $c=1$ boundary state in terms of Kac-Moody generators, we can rewrite the partition function entirely in terms of such generators:

$$
\begin{aligned}
Z^{N B_{V}}= & \sec (\delta / 2)\langle 0| \exp \left[\sum _ { n = 1 } ^ { \infty } \frac { 2 } { n } \left\{\bar{J}_{n}^{X 3}\left(\cos \delta J_{n}^{X 3}-\sin \delta J_{n}^{Y 3}\right)+\right.\right. \\
& \left.\left.\bar{J}_{n}^{Y 3}\left(\cos \delta J_{n}^{Y 3}+\sin \delta J_{n}^{X 3}\right)\right\}\right] q^{L_{0}+\bar{L}_{0}} \exp \left[\sum_{n=1}^{\infty} \frac{2}{n}\left(\bar{J}_{-n}^{X 3} J_{-n}^{X \theta}+\bar{J}_{-n}^{Y 3} J_{-n}^{Y \theta}\right)\right]|0\rangle
\end{aligned}
$$

where the rotated current

$$
J_{n}^{\theta}=\cos \theta J_{n}^{3}+\frac{\sin \theta}{2 i}\left(J_{n}^{+}-J_{n}^{-}\right),
$$

with $\theta=2 \pi V$, contains all the information about the strength of the boundary potential. The commutation relations for the $\mathrm{SU}(2)_{1}$ Kac-Moody algebra suffice to compute the expansion of the partition function in powers of $q$. We obtain

$$
\begin{aligned}
Z^{N B_{V}}=\frac{1}{\cos \delta / 2}\left\{1+(2 \cos \theta \cos \delta) q^{2}+\right. \\
\\
\left.\left(2 \cos ^{2} \delta+\cos ^{2} \theta \cos (2 \delta)+2 \cos \theta \cos \delta\right)\left(q^{2}\right)^{2}+\cdots\right\} .
\end{aligned}
$$


Each term in this expansion is exact in the parameters $V$ and $\delta$ and requires only some $S U(2)_{1}$ algebra for its calculation. To reiterate, this expression is only valid for the discrete field values where $\tan (\delta / 2)$ is an integer.

For the first non-trivial "magic" point, $(\alpha, \beta)=(1 / 2,1 / 2)$, it is possible to obtain a more explicit expression for the partition function. This point is interesting because it corresponds to a flux of $\pi$ through the unit cell of the potential. The rotation by $\delta=\pi / 2$ amounts to the transformation $X_{L} \rightarrow Y_{L}, Y_{L} \rightarrow-X_{L}$. We can commute $e^{i \delta \mathcal{R}_{L}}$ past $L_{0}$ so that it acts on the Neumann boundary. We now make use of the expression of the Neumann boundary state as a sum over the discrete state primaries $\left|j, j_{z}\right\rangle$ (explained in detail in [9]) to evaluate this action:

$$
\begin{aligned}
\langle N| e^{i \frac{\pi}{2} \mathcal{R}_{L}} & =\sum_{j, l}\left\langle j,\left.0\right|_{L} ^{X}\left\langle j,\left.0\right|_{R} ^{X}\left\langle l,\left.0\right|_{L} ^{Y}\left\langle l,\left.0\right|_{R} ^{Y} \sum_{N, M}\{N\}_{L}^{X}\{N\}_{R}^{X}\{M\}_{L}^{Y}\{M\}_{R}^{Y} e^{i \frac{\pi}{2} \mathcal{R}_{L}}\right.\right.\right.\right. \\
& =\sum_{j, l}\left\langle j,\left.0\right|_{L} ^{Y}\left\langle j,\left.0\right|_{R} ^{X}\left\langle l,\left.0\right|_{L} ^{X}\left\langle l,\left.0\right|_{R} ^{Y}(-1)^{l} \sum_{N, M}\{N\}_{L}^{Y}\{N\}_{R}^{X}\{M\}_{L}^{X}\{M\}_{R}^{Y}\right.\right.\right.\right.
\end{aligned}
$$

where $\{N\}$ denotes a combination of Virasoro generators that forms a basis of descendants for a Virasoro module. We note that the operator $\mathcal{R}_{L}$ does not commute with the Virasoro operators of the fields $X$ and $Y$ (it commutes with the sum, of course), but for this particular value of $\delta$ we get $L^{X} \rightarrow L^{Y}, L^{Y} \rightarrow L^{X}$.

The product of $c=1$ boundary states can be similarly expanded using [9]:

$$
\begin{aligned}
|B\rangle^{X}|B\rangle^{Y}=\sqrt{2} \sum_{N^{\prime}, M^{\prime}}\left\{N^{\prime}\right\}_{L}^{X}\left\{N^{\prime}\right\}_{R}^{X}\left\{M^{\prime}\right\}_{L}^{Y}\left\{M^{\prime}\right\}_{R}^{Y} \times \\
\\
\sum_{j^{\prime}, l^{\prime}} \mathcal{D}_{00}^{j^{\prime}}(\theta) \mathcal{D}_{00}^{l^{\prime}}(\theta)\left|j^{\prime}, 0\right\rangle_{L}^{X}\left|j^{\prime}, 0\right\rangle_{R}^{X}\left|l^{\prime}, 0\right\rangle_{L}^{Y}\left|l^{\prime}, 0\right\rangle_{R}^{Y} .
\end{aligned}
$$

The partition function is the sandwich of $q^{L_{0}+\tilde{L}_{0}}$ between (3.10) and (3.11) and can be expanded as

$$
\begin{aligned}
& Z^{N B_{V}}=\sqrt{2} \sum_{j, l, j^{\prime}, l^{\prime}} \delta_{j, j^{\prime}} \delta_{l, l^{\prime}} \delta_{j, l^{\prime}} \delta_{l, j^{\prime}} \sum_{M, N, M^{\prime}, N^{\prime}} \delta_{M, M^{\prime}} \delta_{N, M^{\prime}} \delta_{M, N^{\prime}} \delta_{N, N^{\prime}} \\
& \mathcal{D}_{00}^{j^{\prime}}(\theta) \mathcal{D}_{00}^{l^{\prime}}(\theta)(-1)^{l}\left(q^{2}\right)^{\epsilon(j, N)+\epsilon(l, M)}
\end{aligned}
$$

where $\epsilon(j, N)$ is the weight of the state $\{N\}|j, 0\rangle$. Summing over all the delta functions and remembering the definition

$$
\sum_{M}\left(q^{4}\right)^{\epsilon(j, M)}=\chi_{j}^{V i r}\left(q^{4}\right)=\frac{q^{4 j^{2}}-q^{4(j+1)^{2}}}{q^{\frac{1}{6}} \prod_{1}^{\infty}\left(1-q^{4 n}\right)}
$$


of the Virasoro character, we obtain our final expression for the partition function:

$$
Z^{N B_{V}}=\sqrt{2} \sum_{j=0}^{\infty}(-1)^{j}\left(\mathcal{D}_{00}^{j}(\theta)\right)^{2} \chi_{j}^{V i r}\left(q^{4}\right)
$$

Once again, the boundary potential strength $V$ appears only in the $S U(2)$ rotation angle $\theta=2 \pi V$.

(3.13) is the answer for non-compact $X$ and $Y$, and it can easily be extended to the compact case. If we choose the compactification radius so that the fields $X^{\prime}, Y^{\prime}$ (that appear after rotating the original fields $X, Y)$ are compactified at the $S U(2)$ radius, the compactified partition function can be computed by the same methods as above with the result

$$
\begin{gathered}
Z=\sqrt{2} \sum_{j=0,1 / 2, \ldots} \sum_{m} \mathcal{D}_{-m, m}^{j}(4 \pi V) e^{-i \pi j} \chi_{j}^{V i r}\left(q^{4}\right) \\
=\sqrt{2} \sum_{j=0,1 / 2, \ldots} \chi^{S U(2)}(\hat{\theta}) \chi_{j}^{V i r}\left(q^{4}\right)=\frac{1}{q^{\frac{1}{6}} f\left(q^{4}\right)} \Theta_{3}\left(\frac{\hat{\theta}}{4 \pi} \mid \tau\right)
\end{gathered}
$$

where $\hat{\theta}=4 \pi V+\pi, q=e^{i \pi \tau}=e^{-2 \pi l / T}, f(x)=\prod_{1}^{\infty}\left(1-x^{n}\right)$ and $\Theta_{3}$ is a Jacobi theta function. Apart from the replacement $q^{2} \rightarrow q^{4}$, this is the same as the $c=1$ partition function at the $S U(2)$ radius.

As an interesting application of our results, we may study the effect of magnetic field on the energy levels of DQM. For zero magnetic field the partition function is a square of the $c=1$ partition function which was evaluated in [9] for one Neumann and one dynamical boundary,

$$
Z_{c=1}=\frac{1}{\sqrt{2}} \sum_{j=0}^{\infty} \mathcal{D}_{00}^{j}(2 \pi V) \chi_{j}^{V i r}\left(q^{2}\right)
$$

Transforming to the open string channel, we find

$$
Z_{c=2}=Z_{c=1}^{2}=\frac{1}{w^{1 / 12} f^{2}(w)} \int_{0}^{2 \pi} \frac{d \varphi_{1}}{2 \pi} \int_{0}^{2 \pi} \frac{d \varphi_{2}}{2 \pi} \sum_{k=-\infty}^{\infty} \sum_{r=-\infty}^{\infty} w^{\left(\frac{\gamma_{1}}{4 \pi}+k\right)^{2}+\left(\frac{\gamma_{2}}{4 \pi}+r\right)^{2}}
$$

where $w=e^{-\pi T / l}$ and

$$
\cos \frac{\gamma_{1}}{2}=\cos (\pi V) \cos \varphi_{1}, \quad \cos \frac{\gamma_{2}}{2}=\cos (\pi V) \cos \varphi_{2}
$$


It is interesting to compare this formula with the corresponding partition function at the first "magic" value of magnetic field. Transforming (3.14) to the open string channel, we find

$$
\begin{aligned}
Z^{N B_{V}} & =\frac{1}{w^{1 / 48} f\left(w^{1 / 2}\right)} \frac{1}{\sqrt{2}} \int_{0}^{2 \pi} \frac{d \varphi_{1}}{2 \pi} \int_{0}^{2 \pi} \frac{d \varphi_{2}}{2 \pi} \sum_{k=-\infty}^{\infty} w^{\frac{1}{2}\left(\frac{\gamma}{4 \pi}+k\right)^{2}} \\
& =\frac{1}{w^{1 / 12} f^{2}(w)} \frac{1}{\sqrt{2}} \int_{0}^{2 \pi} \frac{d \varphi_{1}}{2 \pi} \int_{0}^{2 \pi} \frac{d \varphi_{2}}{2 \pi} \sum_{k=-\infty}^{\infty} \sum_{r=-\infty}^{\infty} w^{\frac{1}{2}\left(\frac{\gamma}{4 \pi}+k\right)^{2}+\left(\frac{1}{4}+r\right)^{2}}
\end{aligned}
$$

where

$$
\cos \frac{\gamma}{2}=\sin (2 \pi V) \sin \frac{\varphi_{1}}{2} \sin \frac{\varphi_{2}}{2} .
$$

This indicates that, near the tight-binding limit $(V=1 / 2)$, there are more bands per unit energy for $\delta=\pi / 2$ than for $\delta=0$. This is not surprising because $\delta=\pi / 2$ corresponds to the non-trivial magnetic flux of $\pi$ per unit cell of the square lattice.

\section{Exact Results From Fermionic Representation}

In the preceding section we obtained a simple representation of the boundary state at the magic values of the magnetic field. We then proceeded to calculate various partition functions and found that, while exact results could be obtained, the derivations were somewhat awkward. For the $c=1$ theory, it was found that most results can also be obtained quite simply by mapping the system into a theory of free fermions [18, 14]. It turns out that the same is true in the $c=2$ case. In this section we review the $c=1$ fermionization and show how to extend it to the $c=2$ system at the "magic" points. We will see that at all these points the fermionic theory is essentially the same.

We begin by reviewing the mapping of the $c=1$ theory into a chiral free fermion action [14]. We start with the lagrangian

$$
L=\frac{1}{4 \pi} \int_{0}^{l} d \sigma\left(\partial_{\mu} X\right)^{2}-V \cos X(0)
$$

where the boundary at $\sigma=0$ is dynamical, while the boundary at $\sigma=l$ is free. We introduce chiral bosons in the usual way via $X(\sigma, t)=X_{L}(t+\sigma)+X_{R}(t-\sigma)$. The Neumann boundary conditions at $\sigma=0$ and $\sigma=l$ allow us to replace $X_{L}$ and $X_{R}$ by

a single left-moving field, periodic on the doubled interval: $X_{L}(\sigma+2 l)=X_{L}(\sigma)$. The interaction term at $\sigma=0$ is then replaced by $-\left.V \cos \left(2 X_{L}\right)\right|_{\sigma=0}$. 
In order to fermionize the theory, Polchinski and Thorlacius introduced an auxiliary boson $Y$. A pair of left-moving fermions were defined by

$$
\psi_{1}=C_{1} e^{i\left(Y_{L}-X_{L}\right)}, \quad \psi_{2}=C_{2} e^{i\left(Y_{L}+X_{L}\right)}
$$

where $C_{i}$ are the cocycles necessary to make the fermions anticommuting. The nonpolynomial bosonic interaction term is mapped into

$$
L_{\text {int }}=-\left.\frac{V}{2}\left(\psi_{1}^{\dagger} \psi_{2}+\psi_{2}^{\dagger} \psi_{1}\right)\right|_{\sigma=0} .
$$

There are some subtleties related to projection onto even fermion number, and dividing by the partition function of the free auxiliary boson $Y$, but this mapping into free fermions with quadratic interaction is a nice way to bring out the hidden simplicity of the $c=1$ case.

Something very similar can be done for our $c=2$ system. First consider the case of $\beta=0$ (no magnetic field). The action (2.1) reduces to two independent copies of the $c=1$ theory (4.1). The two chiral fermions may again be introduced via (4.2), except that now $Y$ is a physical field, not an auxiliary field. The $c=2$ interaction term $-V_{X} \cos \left(2 X_{L}\right)-\left.V_{Y} \cos \left(2 Y_{L}\right)\right|_{\sigma=0}$ is still a quadratic in fermion language:

$$
L_{\text {int }}=-\frac{V_{X}}{2}\left(\psi_{1}^{\dagger} \psi_{2}+\psi_{2}^{\dagger} \psi_{1}\right)-\left.\frac{V_{Y}}{2}\left(\psi_{1} \psi_{2}+\psi_{2}^{\dagger} \psi_{1}^{\dagger}\right)\right|_{\sigma=0} .
$$

It is interesting that when we turn on the magnetic field, exactly the same fermionic theory specified by (4.3) arises at all the "magic" points. To show this, consider a string with opposite charges at the two ends interacting with the constant magnetic field. The magnetic interaction (written with Minkowski signature) becomes

$$
L_{m a g}=\frac{\beta}{4 \pi}\left[\left.\left(X \partial_{t} Y-Y \partial_{t} X\right)\right|_{\sigma=0}-\left.\left(X \partial_{t} Y-Y \partial_{t} X\right)\right|_{\sigma=l}\right] .
$$

The vanishing of boundary terms in the variation of the action leads to identical boundary conditions at $\sigma=0$ and $\sigma=l$,

$$
\partial_{\sigma} X-\frac{\beta}{\alpha} \partial_{t} Y=0, \quad \partial_{\sigma} Y+\frac{\beta}{\alpha} \partial_{t} X=0 .
$$

These boundary conditions induce the following relations between left- and right-movers,

$$
\begin{aligned}
& X_{L}-\frac{\beta}{\alpha} Y_{L}=X_{R}+\frac{\beta}{\alpha} Y_{R}, \\
& Y_{L}+\frac{\beta}{\alpha} X_{L}=Y_{R}-\frac{\beta}{\alpha} X_{R} .
\end{aligned}
$$


These conditions allow us to show that $X_{L}$ and $Y_{L}$ are periodic with period $2 l$, and that the right-movers may be eliminated in favor of the left-movers through

$$
\begin{aligned}
& X_{R}=(\cos \delta) X_{L}-(\sin \delta) Y_{L}, \\
& Y_{R}=(\cos \delta) Y_{L}+(\sin \delta) X_{L},
\end{aligned}
$$

where $\delta$ is the rotation angle specified by (3.2). The interaction term then simplifies to

$$
\left.\left.-V_{X} \cos \left(X_{L}+X_{R}\right)-V_{Y} \cos \left(Y_{L}+Y_{R}\right)\right)\left.\right|_{\sigma=0}=-V_{X} \cos \left(2 X_{L}^{\prime}\right)-V_{Y} \cos \left(2 Y_{L}^{\prime}\right)\right)\left.\right|_{\sigma=0}
$$

where the primed fields are defined in (3.3). It is easily checked that the bulk action for $X^{\prime}$ and $Y^{\prime}$ at any "magic" point is normalized identically to that of $X$ and $Y$ at the $\beta=0$ point. Hence, subsequent fermionization gives the same result as the $\beta=0$ theory of (4.3).

It may come as a surprise that all the "magic" points are governed by the same fermionic theory and, therefore, have the same cylinder partition function. This is due to our particularly simple choice of boundary conditions at $\sigma=l$ (recall that we endowed that end of the string with a charge opposite to the one at $\sigma=0$ ). This simplicity is also evident in the boundary state calculation of the cylinder amplitude,

$$
Z^{B_{0} B_{V}}=\sec ^{2}(\delta / 2)\left\langle N\left|e^{-i \delta \mathcal{R}_{L}} q^{L_{0}+\tilde{L}_{0}} e^{i \delta \mathcal{R}_{L}} e^{-H_{\text {pot }}\left(2 X_{L}\right)-H_{p o t}\left(2 Y_{L}\right)}\right| N\right\rangle
$$

Since $\mathcal{R}_{L}$ commutes with $L_{0}$, this reduces to

$$
Z^{B_{0} B_{V}}=\sec ^{2}(\delta / 2)\left(\left\langle\left. N\right|_{X} q^{L_{0}+\tilde{L}_{0}} e^{-H_{p o t}\left(2 X_{L}\right)} \mid N\right\rangle_{X}\right)^{2}
$$

which is simply the square of the $c=1$ partition, except for the factor coming from the magnetic field contribution to the boundary entropy. Thus, at any "magic" point we find the same result as for the completely decoupled $\beta=0$ theory.

As we showed in the previous section, matters are more complicated if the boundary condition at $\sigma=l$ is simply Neumann. Then the magnetic rotation does not cancel, and the cylinder amplitude depends on the "magic" point. It can be evaluated explicitly only in the first non-trivial case $(\beta=\alpha)$. Here we repeat this calculation using the free fermion theory.

Since (4.6) still applies at $\sigma=0$, we may repeat the previous transformations to arrive at the fermion coupling of (4.3). Now separate the Dirac fermions into real and imaginary parts, $\psi_{1}=\chi_{11}+i \chi_{12}, \psi_{2}=\chi_{21}+i \chi_{22}$ so that there are four left-moving Majorana fermions $\chi^{a}=\left(\chi_{11}, \chi_{12}, \chi_{21}, \chi_{22}\right)$ and a corresponding set of right-movers. The boundary 
interaction is a localized Majorana mass term which in general has the effect of rotating the left-movers into right-movers via an $S O(4)$ transformation $\chi^{a} \rightarrow R_{I b}^{a} \chi_{b}$. It is not too hard to show that the boundary interaction (4.3) generates the particular $S O(4)$ rotation

$$
R_{I}=\left(\begin{array}{cccc}
\cos \pi\left(V_{X}-V_{Y}\right) & 0 & -\sin \pi\left(V_{X}-V_{Y}\right) & 0 \\
0 & \cos \pi\left(V_{X}+V_{Y}\right) & 0 & -\sin \pi\left(V_{X}+V_{Y}\right) \\
\sin \pi\left(V_{X}-V_{Y}\right) & 0 & \cos \pi\left(V_{X}-V_{Y}\right) & 0 \\
0 & \sin \pi\left(V_{X}+V_{Y}\right) & 0 & \cos \pi\left(V_{X}+V_{Y}\right)
\end{array}\right)
$$

We should also include the subsequent magnetic rotation of the boundary state, $e^{i \delta \mathcal{R}_{L}}$, which now does not cancel.2 For $\delta=\pi / 2$, the magnetic rotation transforms the bosonic fields according to

$$
X_{L}^{\prime} \rightarrow Y_{L}^{\prime}, \quad Y_{L}^{\prime} \rightarrow-X_{L}^{\prime}
$$

In fermionic language this translates into $\chi^{a} \rightarrow R_{M b}^{a} \chi_{b}$, where

$$
R_{M}=\left(\begin{array}{cccc}
0 & 0 & 1 & 0 \\
0 & 0 & 0 & -1 \\
1 & 0 & 0 & 0 \\
0 & 1 & 0 & 0
\end{array}\right)
$$

The overall rotation of the fermionic boundary state is $R=R_{M} R_{I}$. It should be noted that the magnetic field acts locally on the fermions only at $\delta=\pi / 2$ : at other magic values of the field, the magnetic field rotation will not be a simple $S O(4)$ rotation.

In order to proceed we have to choose the boson compactification radius. The simplest case is if we are at the $\mathrm{SU}(2)$ radius. From the expressions for the fermions, (4.2) we see that the states created by them have momenta $p_{X}^{L}=n, p_{Y}^{L}=m$ with $n+m$ even. In principle we could also consider states with $n+m$ odd. They do not contribute, however; the magnetic field term projects them out because it interchanges $n \leftrightarrow m$ and the potential terms change $n$ and $m$ by two. Using the fermions, the partition function at the $\mathrm{SU}(2)$ radius may be written as

$$
\begin{gathered}
Z_{S U(2)}^{N B_{V}}=\sqrt{2}\left(q^{2}\right)^{-2 / 24} \prod_{n=1}^{\infty} \operatorname{det}\left(I+q^{2 n-1} R\right)= \\
\sqrt{2}\left(q^{2}\right)^{-2 / 24} \prod_{n=1}^{\infty}\left(1-q^{4 n-2}\right)\left(1-2 \sin \pi\left(V_{X}+V_{Y}\right) q^{2 n-1}+q^{4 n-2}\right)
\end{gathered}
$$

2 In open string language this rotation introduces twisted boundary conditions in the $\sigma$ direction. 
which is equal to our previous result (3.14) obtained using the bosonic theory ( with the replacement $\left.V \rightarrow\left(V_{X}+V_{Y}\right) / 2\right)$.

To calculate the partition function for the infinite radius case we should restrict to states with $n=m=0$, i.e. zero fermion number for both $\psi_{1}$ and $\psi_{2}$. We can project onto states having zero fermion numbers by inserting into the partition function a phase $e^{i \varphi_{1} F_{1}+i \varphi_{2} F_{2}}$ and then integrating over $\varphi_{i}$. The insertion of these phases corresponds to an additional $\mathrm{SO}(4)$ rotation

$$
R(\varphi)=\left(\begin{array}{cccc}
\cos \varphi_{1} & \sin \varphi_{1} & 0 & 0 \\
-\sin \varphi_{1} & \cos \varphi_{1} & 0 & 0 \\
0 & 0 & \cos \varphi_{2} & \sin \varphi_{2} \\
0 & 0 & -\sin \varphi_{2} & \cos \varphi_{2}
\end{array}\right)
$$

The overall rotation is given by the matrix $\tilde{R} \equiv R(\varphi) R$, and the partition function becomes

$$
\begin{gathered}
Z_{R=\infty}^{N B_{V}}=\sqrt{2} \int_{0}^{2 \pi} \frac{d \varphi_{1}}{2 \pi} \frac{d \varphi_{2}}{2 \pi}\left(q^{2}\right)^{-2 / 24} \prod_{n=1}^{\infty} \operatorname{det}\left(I+q^{2 n-1} \tilde{R}\right)= \\
\sqrt{2} \int_{0}^{2 \pi} \frac{d \varphi_{1}}{2 \pi} \frac{d \varphi_{2}}{2 \pi}\left(q^{2}\right)^{-2 / 24} \prod_{n=1}^{\infty}\left(1-q^{4 n-2}\right)\left(1+q^{2 n-1}\left(\cos \varphi_{1}-\cos \varphi_{2}\right) \sin 2 \pi V+q^{4 n-2}\right)
\end{gathered}
$$

where we have, for simplicity, set $V_{X}=V_{Y}=V$. We have again verified that this is identical to (3.13), the result obtained using the bosonic theory.

\section{Boundary S-Matrix for Special Magnetic Field Values}

If the boundary is interpreted as an impurity in a bulk system, it is often important to know the S-matrix for scattering from the impurity. As is by now well-understood [16,9], the S-matrix can be constructed directly from the boundary state. The boundary state encodes the information on how the left movers are reflected as right movers. We can extract the S matrix from the correlation function of left moving $\partial X$ and right moving $\bar{\partial} X$ fields. This reflection condition implies that we can express the right movers in terms of 
left movers in the following way 3

$$
\begin{aligned}
\bar{\partial} X \rightarrow \cos \theta \partial X^{\prime}-\cos \theta \tan (\delta / 2) \partial Y^{\prime}+ \\
\sin \theta\left[J^{1}\left(X^{\prime}\right)-\tan (\delta / 2) J^{1}\left(Y^{\prime}\right)\right], \\
\bar{\partial} Y \rightarrow \cos \theta \partial Y^{\prime}+\cos \theta \tan (\delta / 2) \partial X^{\prime}+ \\
\sin \theta\left[J^{1}\left(Y^{\prime}\right)+\tan (\delta / 2) J^{1}\left(X^{\prime}\right)\right],
\end{aligned}
$$

where both sides are evaluated at $\bar{z}$, we use the fields (3.3) and we define

$$
J^{1}\left(X^{\prime}\right)=\frac{e^{-2 i X^{\prime}}-e^{2 i X^{\prime}}}{2}, \quad \theta=2 \pi V .
$$

These formulae enable us to calculate any $S$-matrix element. While the operators $\partial X$ describe localized excitations, we find that, when they scatter off the boundary, solitons, described by the exponentials in (5.1), are created. The discussion of soliton sectors is parallel to that in [9].

In applications to DQM, we are interested in calculating correlation functions of the fields $\dot{X}(t)$ and $\dot{Y}(t)$ at the boundary. These correlation functions can be calculated from the bulk ones by taking the limit

$$
\dot{X}(t)=\lim _{z, \bar{z} \rightarrow t}(\partial X+\bar{\partial} X)
$$

We can calculate in this way all two point correlation functions:

$$
\left\langle\dot{X}\left(t_{1}\right) \dot{X}\left(t_{2}\right)\right\rangle=-2\left[\cos ^{2}(\theta / 2)+\sin ^{2}(\theta / 2) \tan ^{2}(\delta / 2)\right] \frac{1}{\left(t_{1}-t_{2}\right)^{2}},
$$

the same result for $\langle\dot{Y} \dot{Y}\rangle$, and

$$
\left\langle\dot{X}\left(t_{1}\right) \dot{Y}\left(t_{2}\right)\right\rangle=-2 i \cos \theta \tan (\delta / 2) \pi \delta^{\prime}\left(t_{1}-t_{2}\right)
$$

which is a contact term. These correlation functions satisfy the duality relations postulated in [19], relating their behavior at the different "magic" points.

3 This is the naive result of first carrying out the $S O(2)_{L}$ rotation due to the magnetic field and then the independent $S U(2)_{L}$ rotations due to the $c=1$ boundary states. It is correct as long as we are allowed to ignore the commutation phases of the various dimension one operators. As usual, this is true only for the "magic" values of magnetic field. 
Now we consider higher point functions. As usual we will be interested in the connected parts. If we focus our attention on the long time behavior we can simplify the calculations by taking the limit in (5.2) before we calculate the correlators. Using (5.1) we get

$$
\begin{aligned}
& \dot{X}=2\left[\partial X^{\prime}+J^{\theta}\left(X^{\prime}\right)\right]+2 \tan (\delta / 2)\left[\partial Y^{\prime}-J^{\theta}\left(Y^{\prime}\right)\right] \\
& \dot{Y}=2\left[\partial Y^{\prime}+J^{\theta}\left(Y^{\prime}\right)\right]-2 \tan (\delta / 2)\left[\partial X^{\prime}-J^{\theta}\left(X^{\prime}\right)\right]
\end{aligned}
$$

where we have defined

$$
J^{\theta}\left(X^{\prime}\right) \equiv \cos \theta \partial X^{\prime}+\sin \theta J^{1}\left(X^{\prime}\right)
$$

Now we will show that correlation functions of only $\dot{X}_{\mathrm{s}}$ have no connected piece. To that effect we perform a sequence of field redefinitions to get

$$
\begin{gathered}
\dot{X}=N \partial U \\
\dot{Y}=-N\left[(\cos \gamma) J^{1}(W \cos \gamma+U \sin \gamma)+(\sin \gamma) J^{1}(U \cos \gamma-W \sin \gamma)\right]
\end{gathered}
$$

where $U, W$ are uncorrelated free fields with the same normalization as the fields $X^{\prime}, Y^{\prime}$ and we have defined

$$
N=2\left[\cos ^{2}(\theta / 2)+\sin ^{2}(\theta / 2) \tan ^{2}(\delta / 2)\right]^{1 / 2}, \quad \tan \gamma=\tan (\delta / 2) \tan (\theta / 2)
$$

For the case in which we only have $\dot{X}_{\mathrm{s}}$ the higher-point correlation functions reduce to the free correlation functions except for an overall factor.

$$
\left\langle\dot{X}\left(t_{1}\right) \cdots \dot{X}\left(t_{2 n}\right)\right\rangle=N^{2 n}\left\langle\partial U\left(t_{1}\right) \cdots \partial U\left(t_{2 n}\right)\right\rangle
$$

Thus correlation functions of only $\dot{X}_{\mathrm{s}}$ have no connected parts despite the interactions. This is precisely the situation we had for the single field case [9]. The same is, of course, true for correlation functions involving only $\dot{Y}$ s. We have also calculated mixed correlation functions with two $\dot{Y}$ s, finding the following connected piece

$$
\begin{gathered}
\left\langle\dot{X}\left(t_{1}\right) \cdots \dot{X}\left(t_{2 n}\right) \dot{Y}\left(s_{1}\right) \dot{Y}\left(s_{2}\right)\right\rangle_{\text {connected }}=\frac{(-1)^{n+1}}{2} N^{2 n+2} \cos ^{2} \gamma \sin ^{2} \gamma \times \\
{\left[(\sin \gamma)^{2 n-2}+(\cos \gamma)^{2 n-2}\right] \frac{\left(s_{1}-s_{2}\right)^{2 n-2}}{\prod_{1}^{2 n}\left(t_{i}-s_{1}\right)\left(t_{i}-s_{2}\right)}}
\end{gathered}
$$

These quantities have previously been calculated perturbatively by Freed [20] and our results agree with hers up to a factor of 2 . We could go further and calculate more 
general connected correlators, but little new would be learned. If the potential is turned off $(V \rightarrow 0)$ or made infinitely strong $\left(V \rightarrow \frac{1}{2}\right)$, all the connected higher point functions vanish, as expected. What is remarkable is that for intermediate values of the potential strength, the connected n-point functions are, apart from overall constants, simple rational functions of coordinate differences. This is in accord with general arguments by Freed in the work cited above. The momentum-space S-matrix elements are correspondingly piecewise linear functions of the scattering particle energies. Presumably this would not be true at the perturbative fixed points that govern the behavior away from the "magic" magnetic field values. It would be instructive to compute scattering data in those theories.

\section{Conclusions}

In this paper we have studied a class of $c=2$ boundary field theories whose partition function reduces to a one-dimensional Coulomb gas with charges obeying fractional interchange statistics. The typical context for this theory is the dissipative quantum mechanics of a particle moving in a two-dimensional periodic potential and a magnetic field. The size of the magnetic field determines the statistics phase. This phase has a very significant effect on the renormalization group flow of the theory and we have devoted this paper to identifying and exploring the fixed points of these flows.

For certain discrete values of the magnetic field, the statistics phase is unity and it turns out that we can find the exact boundary state describing the associated conformal field theory. It is related in a simple way to the exact boundary state which governs the $c=1$ version of this problem (dissipative quantum mechanics of a particle moving in a periodic one-dimensional potential with no magnetic field). Thus, the boundary state and all other physical quantities are built out of $S U(2)$ group rotations (even though strict $S U(2)$ symmetry is broken by the potential itself). We have derived explicit formulae for partition functions and S-matrix elements at the lowest non-trivial special value of the magnetic field.

When the statistics phase is not unity, we find a renormalization group flow to a nontrivial fixed point that is reliable (perturbative) for suitably chosen $\alpha$ and $\beta$. In these cases we find that there is a nonzero boundary entropy and we can verify that it decreases along the renormalization group flow. It should be feasible and instructive to use open string field theory techniques to compute the resulting zero-temperature entropy as a string field theory action. 
The most promising potential application of the type of boundary field theory we have discussed here is edge current tunneling in the quantum Hall effect. The $c=1$ boundary field theory, in particular, has found spectacular application [2] in the description of edge tunneling in the $\nu=1 / 3$ fractional quantum Hall effect. These applications rely in an essential way on the remarkable fact that the $c=1$ boundary sine-Gordon model flows toward the conformal fixed point through a sequence of integrable theories [21]. It is tempting to speculate that the type of theory discussed in this paper will have similar application to the quantum Hall problem at some other filling factor or more complex geometry. A necessary precondition for this is presumably to find integrable models which flow to our conformal theories. We have not seen how to do this, but we hope that this work will stimulate others to try.

\section{Acknowledgements}

This work was supported in part by DOE grant DE-FG02-91ER40671. The work of I. R. K. was also supported in part by NSF Presidential Young Investigator Grant No. PHY-9157482, James S. McDonnell Foundation Grant No. 91-48 and the A. P. Sloan Foundation. 


\section{References}

[1] I. Affleck and A. Ludwig, Nucl. Phys. B352 (1991) 841.

[2] P. Fendley, A. Ludwig and H. Saleur, cond-mat/9408068

[3] A. O. Caldeira and A. J. Leggett, Physica 121A(1983) 587; Phys. Rev. Lett. 46 (1981) 211; Ann. of Phys. 149 (1983) 374.

[4] C. G. Callan, L. Thorlacius, Nucl. Phys. B329 (1990) 117.

[5] C. G. Callan, C. Lovelace, C. R. Nappi, and S. A. Yost, Nucl. Phys. B293 (1987) 83; Nucl. Phys. B308 (1988) 221.

[6] A. Abouelsaood, C. G. Callan, C. R. Nappi and S. A. Yost, Nucl. Phys. B280 (1987) 599.

[7] E. Fradkin and A. Tseytlin, Phys. Lett. 163B (1985) 123.

[8] C. G. Callan and S. Das, Phys. Rev. Lett 51 (1983) 1155.

[9] C. G. Callan and I. R. Klebanov, Phys. Rev. Lett. 72 (1994) 1968; C. G. Callan, I.

R. Klebanov, A. Ludwig and J. M. Maldacena, Nucl. Phys. B422 (1994) 417.

[10] M. Ya Azbel', Zh. Eksp. Teor. Fiz. 46 (1964) 929; D. R. Hofstadter, Phys. Rev. B14 (1976) 2239; G. H. Wannier, Phys. Status Solidi B88 (1978) 757

[11] C.Callan, A. Felce, D. Freed, Nucl.Phys.B392 (1993) 551.

[12] J. L. Cardy, Nucl. Phys. B324 (1989) 581.

[13] I. Klebanov and L. Susskind, Phys. Lett. B200 (1988) 446.

[14] J. Polchinski and L. Thorlacius, Phys. Rev. D50 (1994) 622.

[15] P. Fendley, F. Lesage and H. Saleur, hep-th/9409176

[16] I. Affleck and A. Ludwig, Phys. Rev. Lett 67 (1991) 161.

[17] C. Callan, C. Lovelace, C. Nappi and S. Yost, Nucl. Phys. B293 (1987) 83.

[18] F. Guinea, V. Hakim and A. Muramatsu, Phys. Rev. Lett. 54 (1985) 263.

[19] C. G. Callan and D. Freed, Nucl. Phys. B374 (1992) 543.

[20] D. Freed, Nucl. Phys. B409 (1993) 565.

[21] S. Ghoshal and A. B. Zamolodchikov, Int. J. Mod. Phys. A9 (1994) 3841. 